\title{
Development of Ultra High Sensitivity UV Silicon Carbide Detectors
}

\author{
Feng Yan ${ }^{1, a}$, Xiaobin Xin ${ }^{2, b}$, Petre Alexandrov ${ }^{3, c}$, Carl M. Stahle ${ }^{4, d}$, Bing \\ Guan $^{1, \mathrm{e}}$, and Jian H. Zhao ${ }^{2, \mathrm{f}}$ \\ ${ }^{1}$ Detector Systems Branch, Code 553, NASA-GSFC/Muniz, Greenbelt, MD 20771 \\ ${ }^{2}$ Dept. of ECE, Rutgers Univ., 94 Brett Road, Piscataway, NJ 08854 \\ ${ }^{3}$ United Silicon Carbide, Inc., 100 Jersey Ave., New Brunswick, NJ08902 \\ ${ }^{4}$ Detector Systems Branch, Code 553, NASA-GSFC, Greenbelt, MD 20771 \\ afyan@pop500.gsfc.nasa.gov, bxinxb@ece.rutgers.edu, cuscalexandrov@unitedsic.com, \\ dCarl.M.Stahle@nasa.gov, ebguan@pop500.gsfc.nasa.gov, fjzhao@ece.rutgers.edu
}

Keywords: detector, detectivity, ultraviolet, avalanche photodiode, photon-counter.

\begin{abstract}
A variety of silicon carbide ( $\mathrm{SiC}$ ) detectors have been developed to study their sensitivity, including Schottky photodiodes, p-i-n photodiodes, avalanche photodiodes (APDs), and single photon-counting APDs. Due to the very wide bandgap and thus extremely low leakage current, SiC photo-detectors show excellent sensitivity. The specific detectivity, $D^{*}$, of SiC photodiodes are many orders of magnitude higher than the $D^{*}$ of other solid state detectors, and for the first time, comparable to that of photomultiplier tubes (PMTs). SiC APDs have also been fabricated to pursue the ultimate sensitivity. By operating the SiC APDs at a linear mode gain over $10^{6}$, single photoncounting avalanche photodiodes (SPADs) in UV have been demonstrated.
\end{abstract}

\section{Introduction}

High sensitivity is always a major goal for photo-detectors. The ultimate sensitivity of a detector can be defined as one over the noise equivalent power (NEP), which corresponds to the case when the signal-to-noise ratio $(S N R)$ is equal to unity, and is often referred as detectivity. However, the $N E P$ increases as the area and bandwidth increase. As an area and bandwidth independent figure of merit, one over NEP normalized to the square root of area and bandwidth is generally used to compare the sensitivity of different types of detectors. This is the specific detectivity or $D^{*}$ and is expressed as [1]:

$$
D^{*}=\frac{\sqrt{A \cdot B}}{P} \cdot S N R=\frac{\sqrt{A \cdot B}}{N E P},
$$

where $A$ is the area, $B$ is the bandwidth, $P$ is the incident power, and $S N R$ is the corresponding signal-to-noise ratio. The higher the $D^{*}$, the more sensitive is the detector. At zero bias when Johnson noise dominates, $D^{*}$ can be rewritten into [2]

$$
D^{*}=\frac{q \eta}{h v} \cdot\left[\frac{R_{o} A}{4 k_{B} T}\right]^{1 / 2},
$$

where $A$ is the detector area, $\eta$ is the quantum efficiency, $h$ is Planck's constant, $v$ is the radiation frequency, $k_{B}$ is the Boltzmann constant, $T$ is the temperature, and $R_{o}$ equals to $(d V / d I)_{V=0}$ with $R_{o} A$ being area independent. Note that $R_{o}$ decreases as dark current decreases and $D^{*}$ increases as $R_{o} A$ increases. $R_{O} A$ is frequently used as an area-independent figure of merit for Johnson noise limited detectors, and a larger value indicates better performance.

$4 \mathrm{H}-\mathrm{SiC}$ has a bandgap about three times wider than that of Si. The dark current can be many orders of magnitude lower than that of $\mathrm{Si}$. Therefore, $\mathrm{SiC}$ detectors can have much higher $D^{*}$ than other detectors. In this paper, we will present the $D^{*}$ of a variety of $\mathrm{SiC}$ detectors, including $4 \mathrm{H}-\mathrm{SiC}$ 
Schottky photodiodes, $p-i-n$ photodiodes, avalanche photodiodes (APDs), and single photon-counting avalanche photodiodes (SPADs).

\section{Results and Discussion}

4H-SiC Schottky photodiodes with the device area up to $5 \mathrm{~mm} \times 5 \mathrm{~mm}$ have been designed and fabricated for UV and extreme UV (EUV) detection. The details of the fabrication have been reported previously [3]. At room temperature, the Schottky photodiodes formed with $\mathrm{Pt}$ contacts show extremely low leakage current, less than $1 \times 10^{-15} \mathrm{~A}$ at zero bias. At zero bias, the $R_{o}$ is $1 \times 10^{14} \Omega$ and $R_{o} A$ is $2.5 \times 10^{13} \Omega \mathrm{cm}^{2}$, which corresponds to more than four orders magnitude improvement in contrast to the typical $R_{o} A$ of $\sim 10^{9} \Omega \mathrm{cm}^{2}$ for $\mathrm{Si}$ photodiodes. The quantum efficiency

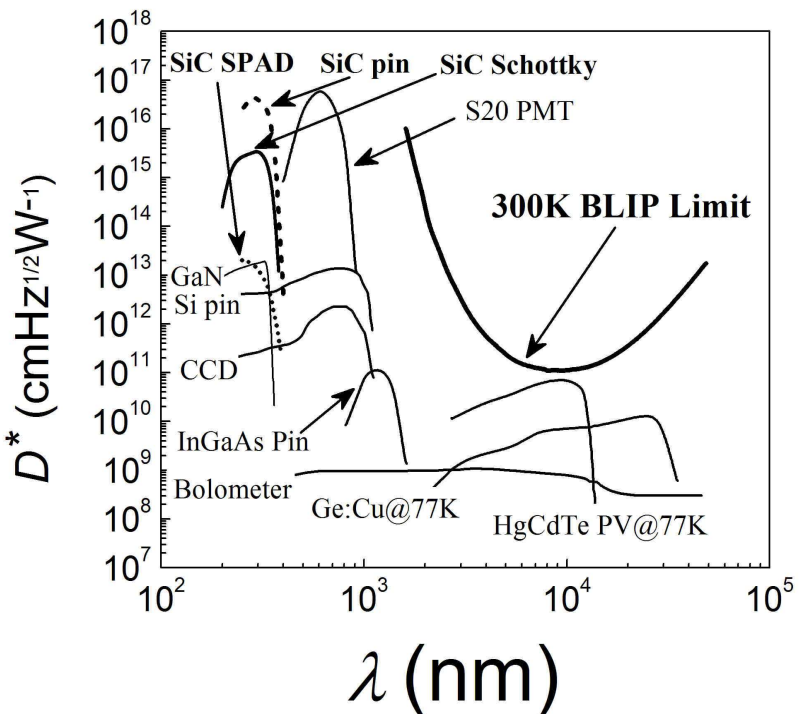

Fig. 1. Comparison of SiC photodiodes fabricated in this work with some common detectors. The $300 \mathrm{~K}$ blackbody radiation limited $D^{*}, 300 \mathrm{~K}$ BLIP limit, is also included as a reference. (QE) varies from $7 \%$ at $130 \mathrm{~nm}$ to $30 \%$ at

250nm. The peak of the $D^{*}$ equals to $3.6 \times 10^{15} \mathrm{cmHz}^{1 / 2} / \mathrm{W}$. By reducing the Pt thickness from $7.5 \mathrm{~nm}$ to less than $3 \mathrm{~nm}$, the absorption in Pt can be further reduced and the QE around 250nm has been increased to $\sim 80 \%$, making the peak $D^{*} \sim 1 \times 10^{16} \mathrm{cmHz}^{1 / 2} / \mathrm{W}$. The $D^{*}(\lambda)$ of the SiC Schottky photodiodes is shown in Fig. 1 and compared with the $D^{*}$ of some common detectors [4].

$4 \mathrm{H}-\mathrm{SiC}$ pin photodiodes have been designed for UV detection and fabricated with the device area of $1.5 \mathrm{~mm} \times 1.5 \mathrm{~mm}$. Figure 2 (a) and (b) show the reverse current density and the UV photoresponse spectra, respectively, and also the results of $\mathrm{Pt} / 4 \mathrm{H}-\mathrm{SiC}$ Schottky photodiodes for comparison. The leakage current of the pin diodes is lower than the noise floor of testing equipment (Keithley $4200 \mathrm{~S}$ ), $1 \times 10^{-14} \mathrm{~A}$, at $-20 \mathrm{~V}$ and below. The current density of pin photodiodes at $-20 \mathrm{~V}$ is 40 times lower than that of Schottky photodiodes. The estimated $R_{o} A$ product at zero bias is $\sim 10^{14} \Omega \mathrm{cm}^{2}$. The peak quantum efficiency is $78 \%$ at $290 \mathrm{~nm}$. The maximum $D^{*}$ is estimated in the mid $10^{16} \mathrm{cmHz}^{1 / 2} / \mathrm{W}$, which is at about the same level as S20 photomultiplier tubes (PMTs). The estimated $D^{*}(\lambda)$ is also shown in Fig. 1 to compare with SiC Schottky photodiodes and other
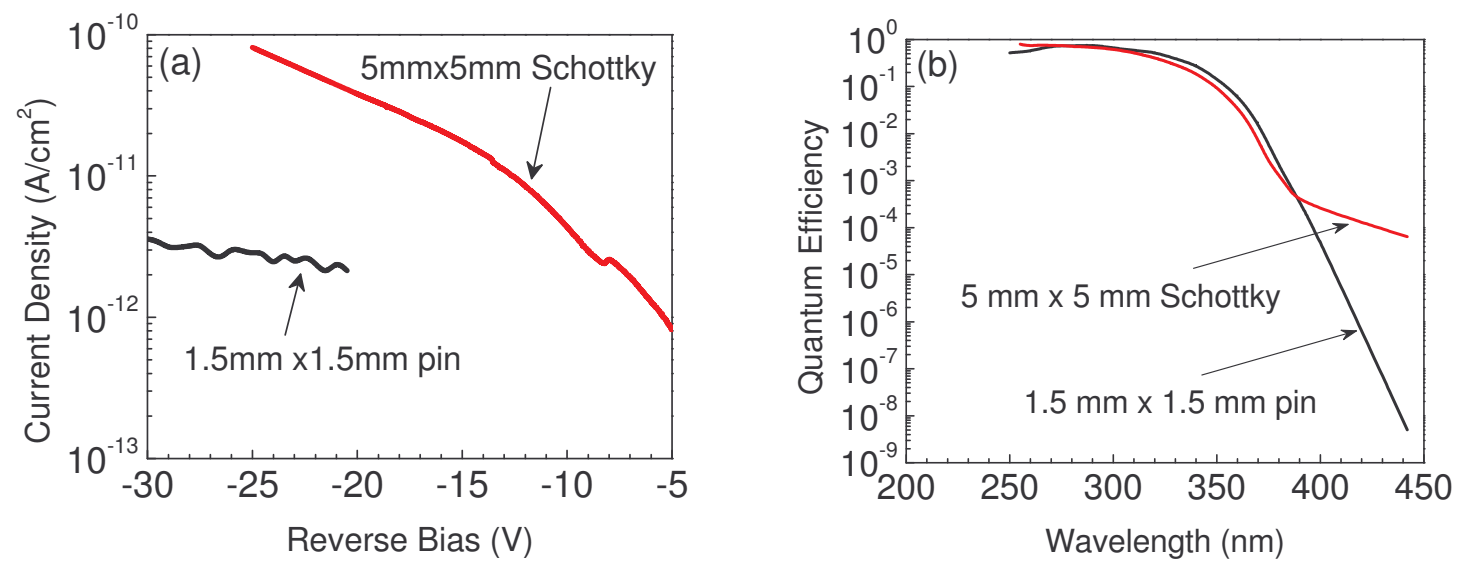

Fig. 2. (a) The leakage current density and (b) quantum efficiency of a $4 \mathrm{H}-\mathrm{SiC}$ SiC pin photodiode and $\mathrm{Pt} / 4 \mathrm{H}-\mathrm{SiC}$ Schottky photodiodes on semilog axes. 
detectors. It should be pointed out that this is the first time that a solid state detector is comparable with PMTs in term of $D^{*}$. In addition, $\mathrm{SiC}$ pin photodiodes have outstanding visible rejection ratio. As shown in Fig. 2b, the QE at 442nm (calibrated using He-Cd laser) was $5.1 \times 10^{-9}$ electron/photon, in contrast to $\sim 10^{-6}$ out of band rejection of PMTs.

As is well-known, the leakage current of pin diodes increases as the intrinsic carrier density $\left(n_{i}\right)$ increases. The $n_{i}$ of $\mathrm{Si}$ is $1.45 \times 10^{10} / \mathrm{cm}^{3}$ at room temperature while $n_{i}$ of $4 \mathrm{H}-\mathrm{SiC}$ is $8 \times 10^{-9} / \mathrm{cm}^{3}$ [5]. The difference is over $10^{18}$ ! The leakage current of Si photodiodes is typically $\sim 10^{-9} \mathrm{~A} / \mathrm{cm}^{2}$. In our study, the lowest leakage current of $4 \mathrm{H}-\mathrm{SiC}$ photodiodes was estimated to be $10^{-16} \mathrm{~A} / \mathrm{cm}^{2}$ which is seven orders of magnitude lower than that of Si photodiodes. However, this measured leakage current of $\mathrm{SiC}$ photodiodes is still over ten orders of magnitude higher than the ideal case. The difference can be reasonably explained by the amount of trap states introduced by the screw dislocations $\left(10^{3} / \mathrm{cm}^{2}\right)$. As the improvement of the material quality continues, the leakage current of $\mathrm{SiC}$ photodiodes will be reduced further by many orders of magnitude, eventually making the $D^{*}$ of SiC photodiodes higher than that of PMTs.

However, a detection system always contains many stages, including at least a photodiode and a set of readout electronics. When the leakage current is very small, the noise of the detector can be much smaller than the readout electronic noise, making the measurement of weak signals very difficult. For example, the most sensitive electrometer on the market is the Keithley model 6430 sub-femtoamp remote source meter, which has a peak-to-peak noise of $0.4 \mathrm{fA}$. This sensitivity is not enough to measure the leakage current of the demonstrated $4 \mathrm{H}-\mathrm{SiC}$ pin photodiodes. In addition, in order to reduce the noise from the readout electronics, the input impedance has to be high enough to suppress the thermal noise of the resistors, $4 k T B / R$, where $k$ is Boltzmann constant, $T$ is temperature, $B$ is bandwidth, and $R$ is the resistance. As a result, the $R C$ time limit becomes intolerably high. For example, the resistance has to be higher than $10^{16}$ to measure the leakage current of $4 \mathrm{H}-\mathrm{SiC}$ pin photodiodes. The resulting $R C$ constant is over $10^{3} \sim 10^{4}$ seconds, which is too long for most applications. Gain at the detector stage is the most effective way to suppress the readout electronics noise and is highly desired to fully take the advantage of the ultra high sensitivity of $\mathrm{SiC}$.

APD is the only approach which can provide high gain without compromising the response speed. 4H-SiC APDs have been demonstrated with a linear mode gain higher than $10^{6}$ at a breakdown voltage $\sim 100 \mathrm{~V}$. The maximum linear gain is over three orders of magnitude higher than that of Si APDs and comparable to the gain of PMTs [6]. A major concern of APDs is the excess noise due to the fluctuation of the multiplication gain, which is roughly proportional to the $k$ value, the ratio of the impact ionization rates of holes and electrons or $\beta / \alpha$. A lower $k$ value reduces the excess noise. $4 \mathrm{H}-\mathrm{SiC}$ APDs have a $k$ value of 0.1, which is similar to Si APDs and much lower than III-V and Ge APDs. Additionally, compared with PMTs, SiC APDs have longer lifetime, smaller size, lower operating voltage, and are inert to magnetic fields. Thus, 4H-SiC APDs can provide a high speed and very sensitive detector solution for many UV and EUV applications. By operating APDs at a gain higher than $10^{6}$, the pulse height generated by a single photon can be significantly higher than the readout electronics noise. As a result, every photon can be converted directly into a pulse. As a result, the photo signal can be directly digitized without requiring any additional analogdigital conversion. This type of APDs is called single photon-counting avalanche diodes (SPADs). For Si and InGaAs/InP SPADs, the devices are required to be operated in Geiger mode for single photon-counting, which sacrifices the response speed and causes severe after-pulsing.

We have operated SiC APDs in linear gain mode at a gain over $10^{6}$ and demonstrated $4 \mathrm{H}-\mathrm{SiC}$ SPADs [7]. Figure 3 shows the photon-counting spectra of a $160 \mu \mathrm{m} \times 160 \mu \mathrm{m}$ SPAD measured in the dark and under UV illumination. The sharp pulses correspond to electrons generated thermally in the dark and by photons under UV illumination. The counting probability of an absorbed photon is $75 \%$ and the dark count rate is $\sim 600 \mathrm{kHz}$. Note that the dark count rate was achieved at room 
temperature and device size is very big. It can be significantly reduced by lowering the operating $\mathrm{T}$ and reducing the size. The $D^{*}(\lambda)$ of the demonstrated $\mathrm{SiC}$ SPADs has been calculated based on Eq. 1 and shown in Fig. 1. The $D^{*}$ of SiC SPADs is lower than SiC pin because the leakage current increased substantially under high stress of the breakdown electric field, but it is expected to be drastically improved as the defect density is further reduced in the future. We believe that $4 \mathrm{H}-\mathrm{SiC}$ APDs and SPADs will perform comparably with commercial PMTs in the near future.

\section{Summary}

In summary, a variety of $4 \mathrm{H}-\mathrm{SiC}$ detectors have been designed, fabricated, and tested. We have demonstrated that $\mathrm{SiC}$ detectors can provide unprecedented high sensitivity and high gain for UV detection. The maximum specific detectivity, $D^{*}$, is greater than $10^{16} \mathrm{cmHz}^{1 / 2} / \mathrm{W}$ for both Schottky and pin photodiodes and is comparable with commercial

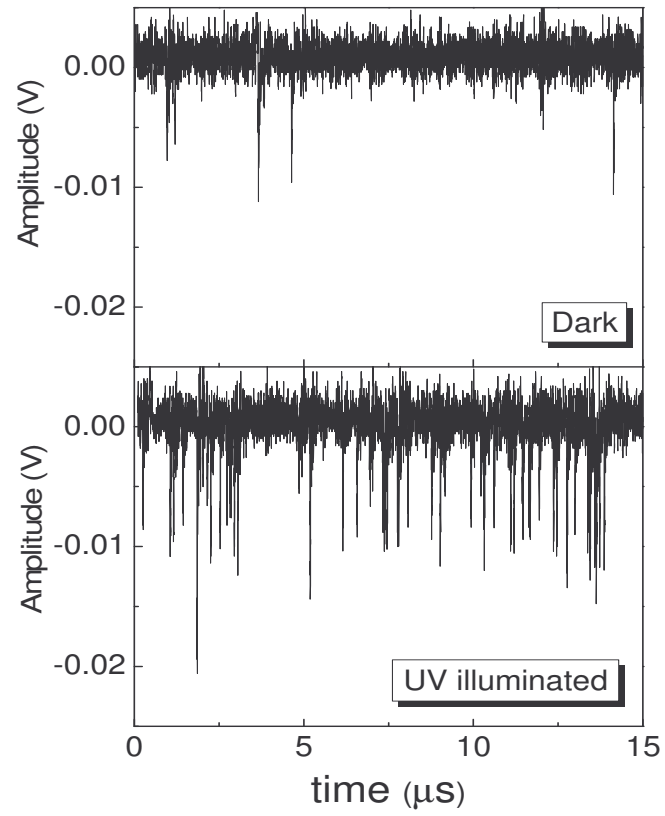

Fig. 3. Photon-counting spectra of a $4 \mathrm{H}-$ $\mathrm{SiC}$ SPAD in dark and under UV illumination. PMTs which is a first for a solid state detector. $4 \mathrm{H}-$ $\mathrm{SiC}$ APDs can provide a higher than $10^{6}$ linear mode gain at an operating voltage $\sim 100 \mathrm{~V}$ and show their potential to compete with PMTs in UV and EUV detection in the future. Single photoncounting capability has been demonstrated by driving the APD over $10^{6}$ multiplication gain at room temperature.

\section{Acknowledgement}

Authors at NASA-GSFC acknowledge the support of this work from the Aeronomy of Ice in the Mesosphere (AIM) and Solar Occultation For Ice Experiments (SOFIE) projects, including Dr. Jim Russell at Hampton University, Christopher Savinell, Joseph Osche, Ronald E. Perison, and William D. Davis at NASA-GSFC, Chad Fish, Jim Peterson, and Dr. John Kemp at Space Dynamics Laboratory of the University of Utah, Dr. Mark Hervig at GATS, Inc., Larry Gordley and Mary Bolton at the Laboratory for Atmospheric and Space Physics at the University of Colorado. Authors at United Silicon Carbide Inc. and SiCLAB acknowledge the financial support provided by NSF (DMI-0339106) through an SBIR Phase I program on SiC SPAD development.

\section{References}

[1] R. J. Keyes, Optical and Infrared Detectors, (New York, Springer-Verlag, 1977), p. 46.

[2] S. L. Chuang, Physics of optoelectronic devices (New York: John Wiley \& Sons, 1995), p. 600.

[3] F. Yan, X. Xin, S. Aslam, Y. Zhao, D. Franz, J. H. Zhao, and M. Weiner: IEEE JQE, Vol. 40 (2004), p. 1315.

[4] The Book of Photon Tools, Stratford: Oriel Instruments, 2003, p. 6.3.

[5] M. E. Levinshtein, et al., Properties of Advanced SemiconductorMaterials GaN, AlN, SiC, BN, SiC, SiGe . (New York, John Wiley \& Sons, Inc., 2001), p. 100.

[6] F. Yan, C. Qin, J. H. Zhao, M. Weiner, B. K. Ng, J. P. David, and R. Tozer: IEE EDL, Vol. 38 (2002), p. 335.

[7] X. Xin, F. Yan, X. Sun, P. Alexandrov, C. M. Stahle, J. Hu, M. Matsumura, X. Li, M. Weiner, and J. H. Zhao: IEE EDL, Vol. 45 (2005), p. 212. 